\title{
Distinguishing black holes from horizonless objects through the excitation of resonances during inspiral
}

\author{
Vitor Cardoso $\odot,{ }^{1,2}$ Adrián del Río, ${ }^{1}$ and Masashi Kimura ${ }^{3}$ \\ ${ }^{1}$ CENTRA, Departamento de Física, Instituto Superior Técnico-IST, Universidade de Lisboa-UL, \\ Avenida Rovisco Pais 1, 1049 Lisboa, Portugal \\ ${ }^{2}$ Theoretical Physics Department, CERN 1 Esplanade des Particules, CH-1211 Geneva 23, Switzerland \\ ${ }^{3}$ Department of Physics, Rikkyo University, Tokyo 171-8501, Japan
}

(Received 11 July 2019; published 22 October 2019)

\begin{abstract}
How good of a description of the dark compact objects in our Universe is the vacuum Kerr geometry? Precision measurements of accreting matter in the deep infrared and gravitational-wave measurements of coalescing objects are finally providing answers to this question. We study the possibility of resonant excitation of the modes of a central object - taken to be very compact but horizonless - during an extrememass-ratio inspiral. We show that, for very compact objects, resonances are indeed excited. However, we find that the impact of such excitation on the phase of the gravitational-wave signal is negligible since resonances are crossed very quickly during inspiral.
\end{abstract}

DOI: 10.1103/PhysRevD.100.084046

\section{INTRODUCTION}

A remarkable feature of classical general relativity is that vacuum spacetime can be curled to the extreme point of producing horizons, the boundaries of causally disconnected regions of spacetime that cloak singularities from faraway observers. Such an extraordinary property requires strong observational evidence for black holes (BHs), a quest that should be placed alongside tests of the equivalence principle. In fact, dark compact horizonless objects are predicted to arise, at a phenomenological level, either when quantum effects are included or when beyond-thestandard model of particle physics is considered $[1,2]$.

Thus far, tests of the $\mathrm{BH}$ nature of compact objects which are based on gravitational-wave (GW) observations rely on (i) small corrections to the GW phase as two compact bodies inspiral, driven by different multipolar structures, tidal deformation, and heating [3-9]; and (ii) echoes of the merger stage, induced by the presence of structure close to the gravitational radius of the final object [10-19]. For a review, see Ref. [1].

Here, we study instead the possibility that the proper modes of oscillation of compact objects are excited and play a role in the inspiraling process. Previous studies focused on a special class of solutions-boson starswhich have a well-defined underlying theory and are of

Published by the American Physical Society under the terms of the Creative Commons Attribution 4.0 International license. Further distribution of this work must maintain attribution to the author(s) and the published article's title, journal citation, and DOI. interest from a particle-physics point of view. Resonant excitation of modes was found to be possible [20]. However, such self-gravitating solutions are never as compact as to be able to mimic the ringdown stage of BHs [1]. Therefore, here we turn to an (artificial) model describing the physics of objects whose surface sits deep down in the gravitational potential. ${ }^{1}$ The compact object is assumed to be spherically symmetric. The exterior is vacuum and therefore described by the Schwarzschild geometry, down to the (hard) surface at

$$
r_{0}=2 M(1+\epsilon) .
$$

We consider both a toy model where a particle coupled to a scalar field orbits the compact object and a more realistic extreme-mass-ratio inspiral driven by GW emission.

\section{SETUP AND RESULTS}

A pointlike mass $m_{0}$ coupled to a scalar field with strength $\gamma$ orbits the central mass $M$ on a circular geodesic of radius $r_{p} \gg M$, emitting scalar and gravitational waves. We consider linearized scalar $(s=0)$ and gravitational $(s=2)$ field perturbations and expand them in Fourier modes of frequency $\omega$. The angular dependence of these fields is separated using spin-s spherical harmonics, labeled with an angular number $\ell$ and an azimuthal number $m$. We used matched asymptotic expansions to solve the relevant

\footnotetext{
${ }^{1}$ Ultracompact objects-so-called gravastars-were investigated in Ref. [21]; it was shown that resonances can be excited during inspiral, but a proper detectability analysis was not performed.
} 
radial wave equations describing the linearized scalar and gravitational field perturbations excited by the particle. The technical details are relegated to the appendixes. We are mostly interested in the possibility of excitation of the internal degrees of freedom of the massive object that could take place when the orbital frequency of the particle approaches a characteristic frequency of the system, and on the possibility that such excitations show up in the detected GW signal. Such excitations of characteristic modes do not take place when the central object is a black hole since the modes of BHs are localized at the light ring, where timelike motion is general unstable [1,22]; consequently, mode excitation by inspiraling bodies can be useful a priori in testing the nature of dark compact objects. We will assume, for simplicity, Dirichlet boundary conditions on the relevant master variables at the surface of the compact object. Gravitational fluctuations will most likely not interact significantly with any putative surface but would cross unimpeded toward the center of the star, where they would be reflected. Thus, these artificial conditions are expected to mimic the physics we want to study.

\section{A. Resonant frequencies}

Assuming reflective boundary conditions on the central object surface, the solution of the relevant wave equations appears as a linear combination of ingoing and outgoing waves at spatial infinity. Given the dissipative character of the system (i.e., energy escaping out to infinity), the mode frequencies $\omega$ are in general complex, and we shall denote their real and imaginary parts as $\omega_{R}$ and $\omega_{I}$; i.e., the characteristic modes are $\omega=\omega_{R}+i \omega_{I}$. When the solution is imposed to be a purely outgoing wave at spatial infinity, corresponding to a maximum of energy flux emitted by the system (i.e., a resonance), we get a condition on the frequencies. This condition defines the quasinormal modes $\omega$ of the system.

Define $\varpi=2 M \omega$. In the small $\epsilon$ regime, we find resonant or quasinormal frequencies at $(n=1,2, \ldots)$,

$$
\begin{aligned}
& \varpi_{s=0} \approx \frac{n \pi}{\log \epsilon}-i \frac{(2 n \pi)^{2 \ell+2} \Gamma(\ell+1)^{6}}{4|\log \epsilon|^{2 \ell+3} \Gamma(2 \ell+1)^{2} \Gamma(2 \ell+2)^{2}}, \\
& \varpi_{s=2} \approx \frac{n \pi}{\log \epsilon}-i \frac{(2 n \pi)^{2 \ell+2} \Gamma(\ell+1)^{2} \Gamma(\ell-1)^{2} \Gamma(\ell+3)^{2}}{8|\log \epsilon|^{2 \ell+3} \Gamma(2 \ell+1)^{2} \Gamma(2 \ell+2)^{2}},
\end{aligned}
$$

where we recall that $\ell$ refers to the spherical harmonic mode. These results agree with previous analytical and numerical studies [1,23].

Now, to excite such quasinormal modes, the angular orbital frequency $\Omega$ of the particle needs to be tuned, $\omega_{R}=m \Omega$. Thus, resonances occur when the radius of the circular orbit is $r_{p}=M^{1 / 3} /\left|\omega_{R} / m\right|^{2 / 3}$ [see Eq. (A7)] with $r_{0} \omega_{R} \simeq n \pi / \log \epsilon$. For the orbit to be stable, the radius should be $r>6 M$, which implies that we focus on

$$
\epsilon<e^{-6 \sqrt{6} n \pi / m} .
$$

\section{B. Fluxes on and off resonance}

In the absence of resonance with the central object, a particle on a circular orbit of radius $r_{p}$ gives rise to an energy flux whose dominant component is

$$
\begin{aligned}
& \dot{E}_{s=0}=\frac{\gamma^{2} m_{0}^{2} M^{2}}{24 \pi^{2} r_{p}^{4}}, \\
& \dot{E}_{s=2}=\frac{32}{5} \frac{m_{0}^{2} M^{3}}{r_{p}^{5}} .
\end{aligned}
$$

These fluxes correspond to the dominant dipolar and quadrupolar modes of the field for $s=0,2$, respectively, and agree with known expressions in the literature (most notably Einstein's quadrupole formula). The structure of the central object is irrelevant in this regime (and thus a central BH would give rise to identical fluxes) [24].

However, when the orbital frequency approaches a resonance frequency, the flux has a sharp peak equal to

$$
\begin{gathered}
\dot{E}_{s=0} \approx \frac{\gamma^{2} m_{0}^{2} m^{4 \ell / 3}}{M^{2} 2^{14 \ell / 3+2}} \frac{\Gamma(2 \ell+2)^{3} \Gamma(2 \ell+1)^{4}\left(\gamma_{\mathrm{EM}}+\psi(\ell+1)\right)^{2}(\log \epsilon)^{10 \ell / 3}}{(n \pi)^{10 \ell / 3} \Gamma(\ell+1)^{12}}, \\
\dot{E}_{s=2} \approx \frac{m_{0}^{2}}{M^{2}} \frac{m^{4 \ell / 3}}{(n \pi)^{10 \ell / 3}}(\log \epsilon)^{10 \ell / 3} \frac{2 \pi \Gamma(2 \ell+2)^{3} \Gamma(2 \ell+1)^{2}(\ell+1)^{4}\left[2 \gamma_{\mathrm{EM}}+\psi(\ell-1)+\psi(\ell+3)\right]^{2}}{2^{2 \ell / 3} \Gamma(-\ell+1 / 2)^{2} \Gamma(\ell+3)^{5} \Gamma(\ell-1)^{3} \Gamma(\ell+1)^{2}},
\end{gathered}
$$

where $\psi(x)=\frac{1}{\Gamma(x)} \frac{d \Gamma(x)}{d x}$ is the digamma function, and $\gamma_{\mathrm{EM}}$ is the Euler-Mascheroni constant. These resonances have a radial width in orbital frequency of $\delta \Omega \sim \omega_{I}$; see Eq. (A51). Notice that, for most parameters of interest and for the dominant modes, $\dot{E}$ at resonance is indeed larger than off resonance.

\section{Impact of resonances on extreme-mass-ratio inspirals}

The pace at which the inspiral proceeds is determinedwithin a quasiadiabatic approach - by energy conservation. An increased flux at resonance implies that the inspiral toward an exotic horizonless object proceeds faster, when 
compared to $\mathrm{BH}$ binaries. In turn, this effect might lead to an observable dephasing in gravitational waveforms. On the other hand, these are very narrow resonances, and thus the accumulated energy release may be small enough that the effect is negligible.

To estimate the impact on the GW phase, one can compute the number of cycles accumulated during the resonant stage. If we denote by $f_{i}$ the orbital frequency of the particle at which a resonance starts, and by $f_{f}$ the orbital frequency at which the corresponding resonance finishes, then $f_{f}-f_{i} \sim \omega_{I} / 2 \pi$ is the resonance width and

$$
N_{\text {res }} \sim \int_{f_{i}}^{f_{f}} \frac{f}{\dot{f}} d f
$$

gives the desired number of cycles. Note that for gravitational waves, $f$ is also half the frequency of the quadrupolar waves being emitted. The variation of the frequency can be computed from the orbital parameters in a quasiadiabatic fashion,

$$
\dot{f} \sim-\frac{3 f}{2 r}\left(\frac{d E_{\mathrm{orb}}}{d r}\right)^{-1} \dot{E} .
$$

Here,

$$
E_{\mathrm{orb}}=m_{0} \frac{1-2 M / r}{\sqrt{1-3 M / r}}
$$

is the gravitational binding energy of the small point particle.

The number of cycles should be compared to those in a BH vacuum spacetime, $N_{\mathrm{BH}}$, obtained by using the flux (4) in the previous expressions (we ignore fluxes through the horizon since these are subdominant [24]). We find, for $l=m=2$ and for the dominant fundamental $n=1$ mode,

$$
\begin{gathered}
N_{\text {res }} \sim 2.4 \times 10^{-11} \frac{10^{-6}}{q}\left(\frac{10}{|\log \epsilon|}\right)^{43 / 3}, \\
\frac{N_{\text {res }}}{N_{\text {BH }}} \sim 9.5 \times 10^{-11}\left(\frac{10}{|\log \epsilon|}\right)^{10},
\end{gathered}
$$

where $q \equiv m_{0} / M$ is the mass ratio. Thus, the small object passes through resonances without any noticeable effect on the GW output. Higher modes are necessarily suppressed even further since Eq. (2) forces $\epsilon$ to decrease exponentially with $n$.

It is, in principle, possible that the number of cycles spent in resonance is small, yet the signal is observable. However, the time $\delta t$ that it takes to cross the resonance can be estimated using $\delta t \sim \omega_{I} / \dot{\Omega}_{\text {orb }}$, with $\dot{\Omega}_{\text {orb }}=$ $d \Omega / d r d r / d E_{\text {orb }} \dot{E}$. We find

$$
\delta t \sim 2.5 \times 10^{-8} \frac{M}{10^{6} M_{\odot}} \frac{10^{-6}}{q}\left(\frac{10}{|\log \epsilon|}\right)^{40 / 3} \mathrm{~s} .
$$

This corresponds to a high-frequency "glitch," inaccessible by current or planned GW detectors.

\section{Discussion}

In conclusion, the inspiral of a small pointlike particle around an ultracompact object can excite the characteristic modes of the central object, which carry important information on the nature of the latter. However, our results indicate that such excitation does not have a significant impact of the phase of the GW, and it leads to only a very high-frequency glitch. Thus, despite initial expectancies, resonant excitation of modes during inspiral turns out not to be a promising mechanism to help constraining the nature of dark ultracompact objects. Our results are based on a simpleminded model for the supermassive object; it would certainly be desirable to extend the analysis to other selfgravitating objects whose surface lies extremely close to the Schwarzschild radius.

\section{ACKNOWLEDGMENTS}

We acknowledge financial support provided under the European Union's H2020 ERC Consolidator Grant "Matter and Strong-Field Gravity: New Frontiers in Einstein's Theory," Grant Agreement No. MaGRaTh-646597. This project has received funding from the European Union's Horizon 2020 research and innovation program under Marie Skłodowska-Curie Grant Agreement No. 690904. We acknowledge financial support provided by FCT/Portugal through Grant No. PTDC/MAT-APL/30043/2017. We acknowledge the SDSC Comet and TACC Stampede2 clusters through NSF-XSEDE Grant No. PHY-090003. The authors would like to acknowledge networking support by GWverse COST Action No. CA16104, "Black Holes, Gravitational Waves and Fundamental Physics."

\section{APPENDIX A: SCALAR CASE}

\section{The setup}

We start with a very simple toy problem, that of a massless scalar field $\Phi$ around a compact horizonless object of mass $M$ in a spacetime background of metric $g_{\mu \nu}$. The scalar field will be excited by introducing a pointlike particle of mass $m_{0}$ coupled to it and orbiting around the central object. The full dynamics is described by the action

$S[g, \Phi]=\int d^{4} x \sqrt{-g}\left(\frac{R[g]}{16 \pi}-g^{\mu \nu} \partial_{\mu} \Phi \partial_{\nu} \Phi^{*}-2 \gamma \Phi T\right)$.

Here, $R[g]$ denotes the Ricci scalar of the metric, $\gamma>0$ is a coupling constant, and $T$ is the trace of the stress tensor of the particle. 
We consider the point particle to be a small perturbation. Thus, the background spacetime is fixed and taken to be described by Schwarzschild exterior geometry, with coordinates $\{t, r, \theta, \phi\}$. All that remains is to solve the scalar field equation of motion coupled to the pointlike particle:

$$
\frac{1}{\sqrt{-g}} \partial_{\mu}\left(\sqrt{-g} g^{\mu \nu} \partial_{\nu} \Phi\right)=\gamma T
$$

For this matter, we expand the fields in Fourier modes of frequency $\omega$ and in spherical harmonics $Y_{\ell m}$ as

$$
\begin{gathered}
\Phi=\sum_{\ell, m} \int \frac{d \omega}{\sqrt{2 \pi}} e^{-i \omega t} \frac{Z_{\ell m}(\omega, r)}{r} Y_{\ell m}(\theta, \phi), \\
r^{2} T=\sum_{\ell, m} \int \frac{d \omega}{\sqrt{2 \pi}} e^{-i \omega t} T_{\ell m}(\omega) Y_{\ell m}(\theta, \phi),
\end{gathered}
$$

where $\ell \geq 0,-\ell \leq m \leq \ell$. For static backgrounds, one finds the equation

$$
\frac{d^{2} Z_{\ell m}}{d r_{*}^{2}}+\left(\omega^{2}-f\left(\frac{\ell(\ell+1)}{r^{2}}+f^{\prime} / r\right)\right) Z_{\ell m}=f \frac{\gamma}{r} T_{\ell m},
$$

with $f=1-2 M / r$, and with $r^{*}$ denoting the tortoise coordinate. The object has a surface at $r=r_{0}$, or in tortoise coordinates $r_{*}=r_{*}^{0}$, where we shall impose reflective conditions.

\section{The source}

If $\tau$ denotes the proper time of the point particle along the world line $z^{\mu}(\tau)=(T(\tau), R(\tau), \vartheta(\tau), \varphi(\tau))$, the corresponding stress-energy tensor is given by

$$
\begin{aligned}
T^{\mu \nu}(x) & =m_{0} \int_{-\infty}^{+\infty} \delta^{(4)}(x-z(\tau)) \frac{d z^{\mu}}{d \tau} \frac{d z^{\nu}}{d \tau} d \tau \\
& =m_{0} \frac{d t}{d \tau} \frac{d z^{\mu}}{d t} \frac{d z^{\nu}}{d t} \frac{\delta(r-R(t))}{r^{2}} \delta^{(2)}(\Omega-\Omega(t)),
\end{aligned}
$$

where the definition of the Dirac delta is taken as $\iiint \int \delta^{(4)}(x) \sqrt{-g} d^{4} x=1$. We shall consider a stable circular geodesic taking place in the equatorial plane. The particle will have an orbit of radius $r=r_{p}>6 M$ and an orbital frequency given by Kepler's law,

$$
\Omega^{2}=\frac{M}{r_{p}^{3}}
$$

Then $g^{\mu \nu} T_{\mu \nu}(x)=\frac{-m_{0}}{\sqrt{-g} U^{t}} \delta\left(r-r_{p}\right) \delta(\theta-\pi / 2) \delta(\phi-\Omega t)$,

where $U^{t}(r)=(1-3 M / r)^{-1 / 2}$.

We can now solve the multipolar moments $T_{\ell m}(\omega)$. Equating Eq. (A4) with Eq. (A8), multiplying both sides by $e^{i \omega^{\prime} t} Y_{\ell^{\prime} m^{\prime}}^{*}$, and integrating on the sphere and in time, we get ${ }^{2}$

$T_{\ell m}(\omega)=-\sqrt{2 \pi} \frac{m_{0} Y_{\ell m}^{*}(\pi / 2)}{U^{t}} \delta\left(r-r_{p}\right) \delta(m \Omega-\omega)$.

\section{The formal solution}

Define two independent solutions of the homogeneous ordinary differential equation (ODE) (A5) as

$$
\begin{gathered}
Z_{1} \sim e^{-i \omega\left(r_{*}-r_{*}^{0}\right)}-e^{i \omega\left(r_{*}-r_{*}^{0}\right)}, \quad r_{*} \rightarrow r_{*}^{0} \\
\sim A_{\text {in }} e^{-i \omega r_{*}}+A_{\text {out }} e^{i \omega r_{*}}, \quad r_{*} \rightarrow+\infty, \\
Z_{2} \sim e^{i \omega r_{*}}, \quad r_{*} \rightarrow+\infty .
\end{gathered}
$$

The former one is considered to have reflective boundary conditions on the central object surface $r_{*}^{0}$, while the latter describes purely outgoing waves at spatial infinity. The two of them are found to be linearly independent by computing their Wronskian, which gives $2 i \omega A_{\text {in }}$. The Green's function reads

$$
G\left(r, r^{\prime}\right)=\frac{\theta\left(r^{\prime}-r\right) Z_{2}\left(r^{\prime}\right) Z_{1}(r)+\theta\left(r-r^{\prime}\right) Z_{2}(r) Z_{1}\left(r^{\prime}\right)}{2 i \omega A_{\text {in }}} .
$$

Then it is easy to show that, at large distances, the inhomogeneous solution is

$$
\begin{aligned}
Z_{\ell m} & =e^{i \omega r_{*}} Z_{\ell m}^{\infty} \delta(m \Omega-\omega) \\
& \equiv-e^{i \omega r_{*}} \frac{\sqrt{2 \pi} m_{0} Y_{\ell m}^{*}(\pi / 2)}{r_{p} U^{t}} \frac{\gamma Z_{1}\left(r_{p}\right)}{2 i \omega A_{\text {in }}} \delta(m \Omega-\omega) .
\end{aligned}
$$

\section{Energy flux}

The energy flux emitted to infinity by the scalar field is determined by

$$
\dot{E}_{s=0} \equiv \frac{d E_{s=0}}{d t}=\lim _{r \rightarrow \infty} \int_{\mathbb{S}^{2}} d \theta d \phi \sqrt{-g} T_{r t}
$$

\footnotetext{
${ }^{2}$ We take the normalization of the spherical harmonics as $\int_{\mathbb{S}^{2}} d \Omega Y_{l m} Y_{\ell^{\prime} m^{\prime}}^{*}=\delta_{l^{\prime} l} \delta_{m^{\prime} m}$. We use the convention $\delta\left(x-x_{0}\right)=$ $\frac{1}{2 \pi} \int e^{i t\left(x-x_{0}\right)} d t$.
} 
where $T_{r t}$ is the relevant component of the scalar stressenergy tensor,

$$
T_{\mu \nu}=\nabla_{\mu} \Phi \nabla_{\nu} \Phi^{*}-\frac{1}{2} g_{\mu \nu} \nabla_{\alpha} \Phi \nabla^{\alpha} \Phi^{*} .
$$

Taking into account the expansion in spherical harmonics (A3) and the asymptotic behavior $Z_{\ell m} \sim Z_{\ell m}^{\infty} e^{i \omega r} \delta(m \Omega-\omega)$ at large radial distances, we find

$$
\begin{aligned}
\frac{d^{2} E_{s=0}}{d t d \Omega}= & \frac{1}{2 \pi} \sum_{\ell^{\prime} m^{\prime}} \sum_{\ell m} \int d \omega d \omega^{\prime} \omega \omega^{\prime} Y_{\ell m} Y_{\ell^{\prime} m^{\prime}} \\
& \times e^{i\left(\omega-\omega^{\prime}\right)(r-t)} Z_{\infty} Z_{\infty}^{\prime} \delta\left(m^{\prime} \Omega-\omega^{\prime}\right) \delta(m \Omega-\omega) .
\end{aligned}
$$

The normalization condition for the spherical harmonics reduces this to

$$
\dot{E}_{s=0}=\frac{1}{2 \pi} \sum_{\ell m}(m \Omega)^{2}\left|Z_{\ell m}^{\infty}\right|^{2} .
$$

\section{Matched asymptotic expansions}

We now want to have an analytical understanding of the solutions of the homogeneous equation at small frequencies. The homogeneous equation can be written in two equivalent forms,

$$
\begin{gathered}
\frac{d^{2} Z}{d r_{*}^{2}}+\left[\omega^{2}-f\left(\frac{\ell(\ell+1)}{r^{2}}+f^{\prime} / r\right)\right] Z=0, \\
\left(r^{2} f(Z / r)^{\prime}\right)^{\prime}+\left[\frac{r^{2}}{f} \omega^{2}-\ell(\ell+1)\right](Z / r)=0,
\end{gathered}
$$

where the primes stand for radial derivatives.

\section{a. The near-region solution}

We follow the procedure in Refs. [23,25]. Consider first a "near region" where $r-r_{0} \ll 1 / \omega$ (we assume $r_{0} \sim 2 M$ to good approximation). Then the second equation above can be written as

$$
x\left(\frac{Z}{r}\right)^{\prime \prime}+\left(\frac{Z}{r}\right)^{\prime}+\left(\frac{\varpi^{2}}{x}-\frac{\ell(\ell+1)}{(1-x)^{2}}\right)\left(\frac{Z}{r}\right)=0,
$$

where the primes are now derivatives with respect to $x \equiv f$, and we introduce the dimensionless frequency $\varpi \equiv \omega r_{H}$. Notice that at the object surface $r=r_{0}, x_{0}=1-r_{H} / r_{0}$, where $r_{H}=2 M$. For $r_{0}=r_{H}(1+\epsilon)$, then $x_{0} \sim \epsilon$.

Defining now $Z / r=x^{i \varpi}(1-x)^{\ell+\epsilon+1} F$, and neglecting $O\left(\varpi^{2}\right)$ and $O(\epsilon)$ terms in the coefficient of $F$, one finds the standard hypergeometric equation,

$x(1-x) \partial_{x}^{2} F+(c-(a+b+1) x) \partial_{x} F-a b F \approx 0$,

with

$a=\ell+\epsilon+1+i 2 \varpi, \quad b=\ell+\epsilon+1, \quad c=1+i 2 \varpi$.

Given that $c$ is not an integer, at around $x=0$, two linearly independent solutions are ${ }_{2} F_{1}(a, b, c, x)$ and $x^{1-c}{ }_{2} F_{1}(a-c+1, b-c+1,2-c, x)$. The general solution in the near region is then

$$
\begin{aligned}
Z= & A x^{-i \varpi}(1-x)^{\ell+\epsilon} F(a-c+1, b-c+1,2-c, x) \\
& +B x^{i \varpi}(1-x)^{\ell+\epsilon} F(a, b, c, x) .
\end{aligned}
$$

For BHs, one imposes boundary conditions corresponding to purely ingoing waves at the horizon, and that implies $B=0$. For "exotic compact objects" (ECOs) with Dirichlet $\mathrm{BCs}$ at the surface $x \sim 0, Z=A x_{0}^{-i \varpi}+B x_{0}^{i \varpi}$, and thus $B=-A x_{0}^{-2 i \varpi}$.

To understand the far-region behavior of the above solution, we use the transformation properties of hypergeometric functions,

$$
\begin{aligned}
F(a, b, c, z)= & \frac{\Gamma(c) \Gamma(c-a-b)}{\Gamma(c-a) \Gamma(c-b)} F(a, b, a+b-c+1,1-z) \\
& +(1-z)^{c-a-b} \frac{\Gamma(c) \Gamma(a+b-c)}{\Gamma(a) \Gamma(b)} F(c-a, c-b, c-a-b+1,1-z) .
\end{aligned}
$$

Therefore, the large $r$ behavior is

$$
\begin{aligned}
Z \sim & \frac{r_{H}^{\ell} \Gamma(-1-2 \ell-2 \epsilon)}{r^{\ell} \Gamma(-\ell-\epsilon)}\left[\frac{A \Gamma(1-2 i \varpi)}{\Gamma(-\ell-\epsilon-2 i \varpi)}+\frac{B \Gamma(1+2 i \varpi)}{\Gamma(-\ell-\epsilon+2 i \varpi)}\right] \\
& +\frac{r^{\ell+1} \Gamma(1+2 \ell+2 \epsilon)}{r_{H}^{\ell+1} \Gamma(\ell+\epsilon+1)}\left[\frac{B \Gamma(1+2 i \varpi)}{\Gamma(\ell+\epsilon+1+2 i \varpi)}+\frac{A \Gamma(1-2 i \varpi)}{\Gamma(\ell+\epsilon+1-2 i \varpi)}\right] .
\end{aligned}
$$




\section{b. The far-region solution}

In the far region (i.e., when $r \gg r_{0}$ ), the wave equation reduces to

$$
\partial_{r}^{2} Z+\left(\omega^{2}-\ell(\ell+1) / r^{2}\right) Z=0
$$

with solutions

$$
Z=r^{1 / 2}\left(\alpha J_{\ell+1 / 2}(\omega r)+\beta J_{-\ell-1 / 2}(\omega r)\right)
$$

which for small $r$ reduces to

$$
Z \sim \alpha \frac{\omega^{\ell+1 / 2} r^{\ell+1}}{2^{\ell+1 / 2} \Gamma(\ell+3 / 2)}+\beta \frac{2^{\ell+1 / 2} r^{-\ell}}{\omega^{\ell+1 / 2} \Gamma[-\ell+1 / 2]} .
$$

\section{c. Matching}

From the behavior of the Bessel functions at $r \rightarrow \infty$, the solution (A27) is asymptotic to

$$
Z \sim e^{i\left(\omega r+\frac{\pi}{2} \ell\right)} \sqrt{\frac{2}{\pi \omega}} \frac{\beta-i \alpha(-1)^{\ell}}{2}+(i \rightarrow-i)
$$

and by now demanding the behavior (A11), we get

$$
A_{\text {in }}=\frac{e^{-i \ell \pi / 2}\left(\beta+i \alpha e^{i \ell \pi}\right)}{\sqrt{2 \pi \omega}} .
$$

Now we want to express $\alpha$ and $\beta$ in terms of the boundary parameters $A, B$. In order to do this, we proceed to match the small $r$ behavior of the far-region solution to the large $r$ behavior of the near-region solution.

First, we use the result

$$
\frac{\Gamma(-2 \ell-1-2 \epsilon)}{\Gamma(-\ell-\epsilon)}=\frac{(-1)^{\ell+1} \Gamma(\ell+1)}{2 \Gamma(2 \ell+2)}+O(\epsilon) .
$$

On the other hand, we can also expand the Gamma function to find

$$
\frac{\Gamma(\ell-\epsilon-2 i \varpi)}{\Gamma(-\ell-\epsilon-2 i \varpi)}=\frac{(\epsilon+2 i \varpi)(-1)^{\ell+1}}{\ell-\epsilon-2 i \varpi} \prod_{k=1}^{\ell}\left(k^{2}+(\epsilon+2 i \varpi)^{2}\right) .
$$

Then, in the limit in which $\varpi \ll 1$, we get

$$
\begin{gathered}
\alpha \approx \frac{2^{\ell+1 / 2}(A+B) \Gamma(\ell+3 / 2) \Gamma(2 \ell+1)}{\varpi^{\ell+1 / 2} \sqrt{r_{H}} \Gamma(\ell+1)^{2}}, \\
\beta \approx \frac{i(A-B) \varpi^{\ell+3 / 2} \ell ! \Gamma(-\ell+1 / 2) \Gamma(\ell+1)}{2^{1 / 2+\ell} \sqrt{r_{H}}(2 \ell+1) !} .
\end{gathered}
$$

Finally, we can write

$$
A_{\mathrm{in}} \approx i \frac{\varpi^{2 \ell+2}(A-B) \Gamma(-\ell+1 / 2) \Gamma(\ell+1)^{5} 2^{-1}+e^{i \ell \pi} \ell \sqrt{\pi}(A+B) \Gamma(2 \ell) \Gamma(2 \ell+2)^{2}}{(2 i)^{\ell} \varpi^{\ell+1} \sqrt{\pi} \Gamma(\ell+1)^{3} \Gamma(2 \ell+2)} .
$$

\section{The black hole flux formulas}

As mentioned before, for BHs, $B=0$. If we focus on the small-frequency regime $\varpi \ll 1$, the second term in Eq. (A27) is suppressed against the first, and [26]

$$
Z\left(r_{p}\right) \approx \alpha \sqrt{r_{p}} J_{\ell+1 / 2}\left(\omega r_{p}\right) \sim \alpha \sqrt{r_{p}} \frac{\left(\omega r_{p}\right)^{\ell+1 / 2}}{2^{\ell+1 / 2} \Gamma(\ell+3 / 2)} .
$$

From Eqs. (A17) and (A13), we find

$$
\dot{E}_{s=0} \approx \sum_{\ell m} \frac{m_{0}^{2} \gamma^{2}\left|Y_{\ell m}\left(\frac{\pi}{2}, 0\right)\right|^{2}}{4 r_{p}^{2}\left|A_{\text {in }}\right|^{2}}\left|Z_{1}\left(r_{p}\right)\right|^{2},
$$

where we use $\omega=m \Omega$.
We can explicitly write $Y_{\ell m}(\pi / 2,0)$ using

$$
\begin{aligned}
Y_{\ell m}(\pi / 2,0)= & \sqrt{(\ell+m) !(\ell-m) !} \\
& \times \frac{(-1)^{(\ell+m) / 2}\left(1+(-1)^{\ell+m}\right) \sqrt{1+2 \ell}}{2^{2+\ell} \sqrt{\pi}((\ell+m) / 2) !((\ell-m) / 2) !} .
\end{aligned}
$$

For definiteness, focus on the $m=\ell$ modes, for which $Y_{\ell \ell}(\pi / 2,0)=(-1)^{\ell} /\left(2^{\ell} \ell !\right) \sqrt{(2 \ell+1) ! /(4 \pi)}$. Using now the expressions for $A_{\text {in }}$ and $\alpha$, in the limit in which $\varpi \ll 1$,

$$
\dot{E}_{s=0} \approx \frac{m_{0}^{2} \gamma^{2}}{4 \pi} \frac{\omega^{2 \ell+2} r_{p}^{2 \ell}}{\Gamma(2 l+2)} .
$$

Using Kepler's law (A7), $\omega=\ell \Omega$, this can also be expressed as 


$$
\dot{E}_{s=0} \approx \frac{m_{0}^{2} \gamma^{2}}{4 \pi} \frac{\ell^{2 \ell+2} M^{\ell+1}}{r_{p}^{\ell+3} \Gamma(2 \ell+2)} .
$$

In particular, for $\ell=m=1$, one finds that

$$
\dot{E}_{s=0} \approx \frac{\gamma^{2} m_{0}^{2} M^{2}}{24 \pi^{2} r_{p}^{4}}
$$

This result agrees very well with full numerical solutions of the inhomogeneous wave equation (at a radius $r_{p}=50 \mathrm{M}$, the simple analytical formula yields the correct result with $8 \%$ accuracy).

\section{The QNMs of ECOs}

For ECOs, we have $B=-A x_{0}^{-2 i \varpi}$. The energy flux is determined by Eq. (A17) with Eq. (A13). We expect resonances then at the poles of Eq. (A34). These are the quasinormal mode (QNM) frequencies of these objects $[1,23]$.

We compute the QNM frequencies as the roots of $A_{\text {in }}(\varpi)=0$. By setting Eq. (A34) equal to zero, we can write

$$
e^{i 2 \varpi \log x_{0}} R(\varpi)=1, \quad R(\varpi)=\frac{1-F_{s=0}(\ell) \varpi^{2 \ell+2}}{1+F_{s=0}(\ell) \varpi^{2 \ell+2}},
$$

with

$$
F_{s=0}(\ell)=\frac{\Gamma(1 / 2-\ell) \Gamma(\ell+1)^{5}}{2 \ell \Gamma(2 \ell) \Gamma(2 \ell+2)^{2} \sqrt{\pi}(-1)^{\ell+1}} .
$$

We proceed to solve the equation iteratively by solving $e^{i 2 \varpi_{i+1} \log x_{0}} R\left(\varpi_{i}\right)=1$ for $\varpi_{i+1}$, with the initial input $\varpi_{0}=0$. In the first iteration, one obtains
TABLE I. The value of QNM frequencies $\omega_{\mathrm{QNM}} \times r_{H}$ for a scalar field and for an ultracompact object with $\epsilon=10^{-6}$.

\begin{tabular}{lll}
\hline \hline$\ell$ & Approximate formula & Numerical calculation \\
\hline 0 & $0.237-7.486 \times 10^{-3} i$ & $0.220-8.646 \times 10^{-3} i$ \\
1 & $0.237-1.075 \times 10^{-5} i$ & $0.260-4.113 \times 10^{-5} i$ \\
2 & $0.237-2.471 \times 10^{-9} i$ & $0.284-2.590 \times 10^{-8} i$ \\
\hline \hline
\end{tabular}

$$
\varpi_{1}=\frac{n \pi}{\left|\log x_{0}\right|}
$$

for all $n \in \mathbb{Z}$. The second iteration gives (since $x_{0}<1$, the logarithm is negative)

$$
\varpi_{2}=\frac{n \pi}{\left|\log x_{0}\right|}-i \frac{1}{2\left|\log x_{0}\right|} \log \left[\frac{1-F_{s=0}(\ell)\left(\frac{n \pi}{\log x_{0}}\right)^{2 \ell+2}}{1+F_{s=0}(\ell)\left(\frac{n \pi}{\log x_{0}}\right)^{2 \ell+2}}\right] .
$$

As argued in the main text, $x_{0} \ll e^{-6 \sqrt{6} n \pi / m}$, so $\left|\log x_{0}\right| \gg n \pi$, and we can approximate

$$
\varpi_{2} \approx \frac{n \pi}{\left|\log x_{0}\right|}+i \frac{F_{s=0}(\ell)(n \pi)^{2 \ell+2}}{\left|\log x_{0}\right|^{2 \ell+3}} .
$$

$F_{s=0}(\ell)$ can be further simplified, and then the next-toleading order result for the QNMs is

$$
\varpi_{s=0} \simeq \frac{n \pi}{\left|\log x_{0}\right|}-i \frac{(2 n \pi)^{2 \ell+2}(\ell !)^{6}}{4(1+2 \ell)^{2}((2 \ell) !)^{4}} \frac{1}{\left|\log x_{0}\right|^{3+2 \ell}} .
$$

The presence of a nonvanishing imaginary part with the correct sign accounts for the exponential decay of the mode in time (stability). This analytical prediction works reasonably well, as checked with the numerical implementation (see Table I).

\section{The ECO flux formulas}

For $B=-A x_{0}^{-2 i \varpi}$, Eq. (A34) gives

$$
\left|A_{\text {in }}\right|^{2} \approx|A|^{2} \frac{\varpi^{4 \ell+4} \cos ^{2}\left(\varpi \log x_{0}\right) \Gamma(1 / 2-\ell)^{2} \Gamma(\ell+1)^{10}+4 \ell^{2} \pi \sin ^{2}\left(\varpi \log x_{0}\right) \Gamma(2 \ell)^{2} \Gamma(2 \ell+2)^{4}}{4^{\ell} \varpi^{2 \ell+2} \pi \Gamma(\ell+1)^{6} \Gamma(2 \ell+2)^{2}},
$$

where we neglected the imaginary part of $\varpi$ to write $\left|1+x_{0}^{2 i \varpi}\right|^{2}=2+2 \cos \left(2 \varpi \log x_{0}\right)=4 \cos ^{2}\left(\varpi \log x_{0}\right)$ and $\left|-1+x_{0}^{2 i \varpi}\right|^{2}=2-2 \cos \left(2 \varpi \log x_{0}\right)=4 \sin ^{2}\left(\varpi \log x_{0}\right)$.

On the other hand, notice from Eq. (A32) that the leading order behavior of $\alpha$ is $\sim\left(1-x_{0}^{-2 i \varpi}\right)$, but around QNM frequencies this is highly suppressed. ${ }^{3}$ Hence we need to go back to the exact expression for $\alpha$,

\footnotetext{
${ }^{3}$ It is proportional to $\operatorname{Im} \varpi \log x_{0}$, and according to Eq. (A43), it is tiny.
}

$$
\begin{aligned}
\alpha= & \frac{2^{\ell+1 / 2} \Gamma(\ell+3 / 2) \Gamma(2 \ell+1)}{\varpi^{\ell+1 / 2} \sqrt{r_{H}} \Gamma(\ell+1)^{2}} \\
& \times\left[\frac{B \Gamma(1+2 i \varpi)}{\Gamma(\ell+1+2 i \varpi)}+\frac{A \Gamma(1-2 i \varpi)}{\Gamma(\ell+1-2 i \varpi)}\right],
\end{aligned}
$$

and work out the subsequent leading order behavior. This will dominate $Z_{1}\left(r_{p}\right)$, in contrast to the $\mathrm{BH}$ case. Doing this, and neglecting the $\beta$ contribution again, 


$$
\left|Z_{1}\left(r_{p}\right)\right|^{2} \approx|\alpha|^{2} \frac{\omega^{2 \ell+1} r_{p}^{2 \ell+2}}{2^{2 \ell+1} \Gamma(\ell+3 / 2)^{2}}=\left(\frac{r_{p}}{r_{H}}\right)^{2 \ell+2} \frac{16|A|^{2} \cos ^{2}\left(\varpi \log x_{0}\right) \varpi^{2} \Gamma(2 \ell+1)^{2}\left(\gamma_{\mathrm{EM}}+\psi(\ell+1)\right)^{2}}{\Gamma(\ell+1)^{4}},
$$

where we introduced the function $\psi(x)=\Gamma^{\prime}(x) / \Gamma(x)$ and the Euler-Mascheroni constant $\gamma_{\mathrm{EM}}$. Using Eq. (A35), near these QNM frequencies, the leading order flux reads

$$
\dot{E}_{s=0} \approx\left(\frac{r_{P}}{r_{H}}\right)^{2 \ell} \frac{\gamma^{2} m_{0}^{2} \varpi^{2 \ell+4} \cos ^{2}\left(\varpi \log x_{0}\right)\left(\gamma_{\mathrm{EM}}+\psi(\ell+1)\right)^{2} \Gamma(2 \ell+1)^{2} \Gamma(2 \ell+2)^{3}}{r_{H}^{2}\left(\varpi^{4 \ell+4} \cos ^{2}\left(\varpi \log x_{0}\right) \Gamma(-\ell+1 / 2)^{2} \Gamma(\ell+1)^{10}+4 \ell^{2} \pi \Gamma(2 \ell)^{2} \Gamma(2 \ell+2)^{4} \sin ^{2}\left(\varpi \log x_{0}\right)\right)} .
$$

At the QNM frequencies, the flux is maximum and yields

$$
\dot{E}_{s=0} \approx \frac{\gamma^{2} m_{0}^{2} m^{4 \ell / 3}}{M^{2} 2^{14 \ell / 3+2}} \frac{\Gamma(2 \ell+2)^{3} \Gamma(2 \ell+1)^{4}\left(\gamma_{\mathrm{EM}}+\psi(\ell+1)\right)^{2}(\log \epsilon)^{10 \ell / 3}}{(n \pi)^{10 \ell / 3} \Gamma(\ell+1)^{12}},
$$

where we used the orbital parameters to excite the resonance at Eq. (A41).

\section{Resonance widths}

Given the Kepler relation (A7), the energy flux (A46) is a function of the radial distance only. To find the width of the ECO resonances, we look for a value $r$ of the radial distance that fulfills $\dot{E}(r)=\frac{1}{2} \dot{E}\left(r_{\mathrm{QNM}}\right)$, where $r_{\mathrm{QNM}}$ denotes the value that gives the resonant frequencies $\varpi_{\mathrm{QNM}}$ written in Eq. (A41), i.e., $\varpi_{\mathrm{QNM}}^{2} / m^{2}=4 M^{3} / r_{\mathrm{QNM}}^{3}$.

We assume that the frequency band will be small, so that we can expand the function $\varpi(r) / m=\sqrt{4 M^{3} / r^{3}}$ in Taylor series at $r_{\mathrm{QNM}}$ as

$\varpi(r)=\varpi_{\mathrm{QNM}}\left[1-\frac{3}{2} \frac{r-r_{\mathrm{QNM}}}{r_{\mathrm{QNM}}}+\frac{15}{4}\left(\frac{r-r_{\mathrm{QNM}}}{r_{\mathrm{QNM}}}\right)^{2}\right]+\ldots$
The scalar flux (A46) can be written now as

$$
\begin{aligned}
\dot{E}_{s=0}(r) & \\
& =\frac{a_{\ell m} \varpi^{2 \ell / 3+4}(r) \cos ^{2}\left(\varpi(r) \log x_{0}\right)}{b_{\ell} \varpi^{4 \ell+4}(r) \cos ^{2}\left(\varpi(r) \log x_{0}\right)+c_{\ell} \sin ^{2}\left(\varpi(r) \log x_{0}\right)},
\end{aligned}
$$

with $\quad b_{\ell} \equiv \Gamma(-\ell+1 / 2)^{2} \Gamma(\ell+1)^{10}>0 \quad$ and $\quad c_{\ell} \equiv$ $4 \ell^{2} \pi \Gamma(2 \ell)^{2} \Gamma(2 \ell+2)^{4}>0$ ( $a_{\ell m}$ will not be needed). The flux peaks at $r_{\mathrm{QNM}}$ and gives $\dot{E}_{s=0}\left(r_{\mathrm{QNM}}\right)=$ $\frac{a_{\ell_{m}}}{b_{\ell}} \varpi_{\mathrm{QNM}}^{-10 / 3 \ell}$. By expanding the energy flux up to second order, we get ${ }^{4}$

$$
\frac{\dot{E}_{s=0}(r)}{\dot{E}_{s=0}\left(r_{\mathrm{QNM}}\right)}=\left[1+5 \ell \frac{r-r_{\mathrm{QNM}}}{r_{\mathrm{QNM}}}+\left(\frac{r-r_{\mathrm{QNM}}}{r_{\mathrm{QNM}}}\right)^{2}\left(\frac{-35 \ell+50 \ell^{2}}{4}-\frac{9 c_{\ell} n^{2} \pi^{2} \varpi_{\mathrm{QNM}}^{-4-4 \ell}}{4 b_{\ell}}\right)\right]+O\left(\frac{r-r_{\mathrm{QNM}}}{r_{\mathrm{QNM}}}\right)^{3} .
$$

Demanding now $\dot{E}_{s=0}(r)=\frac{1}{2} \dot{E}_{s=0}\left(r_{\mathrm{QNM}}\right)$, one gets a quadratic equation for $\left(r-r_{\mathrm{QNM}}\right) / r_{\mathrm{QNM}} \equiv y$,

$$
y^{2}\left[\frac{-35 \ell+50 \ell^{2}}{4}-\frac{9 c_{\ell} n^{2} \pi^{2} \varpi_{\mathrm{QNM}}^{-4-4 \ell}}{4 b_{\ell}}\right]+y(5 \ell)+\frac{1}{2}=0,
$$

which leads to two solutions, $r_{+}$and $r_{-}$, corresponding to the outer and inner radii of the width, respectively. The solution, in the approximation in which $\varpi_{\mathrm{QNM}} \ll 1$ [recall Eq. (A43)], takes the simple form

\footnotetext{
${ }^{4}$ That the coefficient in front of $r-r_{\mathrm{QNM}}$ is not identically zero is just a residue due to the approximations. In an exact approach, this term would not appear. It does not play any role in what follows.
}

$$
y_{ \pm} \approx \pm \frac{\varpi_{\mathrm{QNM}}^{2 \ell+2}}{n \pi} \sqrt{\frac{2 b_{\ell}}{9 c_{\ell}}}
$$

From Eq. (A40), it can be readily checked that $\sqrt{\frac{b_{\ell}}{c_{\ell}}}=F_{s=0}(\ell)(-1)^{\ell+1}$. Thus, we finally arrive at

$$
\begin{aligned}
\left|\frac{r_{+}-r_{-}}{r_{\mathrm{QNM}}}\right| & \approx \sqrt{\frac{8}{9} \frac{\operatorname{Im} \varpi_{\mathrm{QNM}}}{\operatorname{Re} \varpi_{\mathrm{QNM}}}} \\
& \approx \sqrt{\frac{8}{9}} \frac{(2 n \pi)^{2 \ell+1} \Gamma(\ell+1)^{6}}{2\left|\log x_{0}\right|^{2 \ell+2}(2 \ell+1)^{2} \Gamma(2 \ell+1)^{4}},
\end{aligned}
$$


where we used the form (A42). The width in frequency around the value $\varpi_{\mathrm{QNM}}$ can be inferred from Eqs. (A48) and (A50). It reads

$$
\begin{aligned}
2\left|\varpi_{\mathrm{QNM}}-\varpi\left(r_{+}\right)\right| & \approx 3 \varpi_{\mathrm{QNM}} \frac{\left|r_{+}-r_{\mathrm{QNM}}\right|}{r_{\mathrm{QNM}}} \\
& \approx \sqrt{2} \operatorname{Im} \varpi_{\mathrm{QNM}} .
\end{aligned}
$$

\section{APPENDIX B: GRAVITATIONAL CASE}

\section{The setup}

We consider gravitational radiation within the framework of metric perturbations around the Schwarzschild geometry. These perturbations are excited by the inspiral of a small mass around the Schwarzschild BH. We will study these perturbations using the Newman-Penrose formalism combined with the Regge-Wheeler analysis, following Ref. [27].

The fundamental perturbation field is the Weyl scalar $\Psi_{4}$, which is well adapted to analyze outgoing GWs. We expand it as

$$
r^{4} \Psi_{4}=\sum_{\ell, m} \int_{-\infty}^{\infty} d \omega R_{\omega \ell m}(r) e_{-2}^{-i \omega t} Y_{\ell m}(\theta, \phi),
$$

where ${ }_{-2} Y_{\ell m}$ denote the spherical harmonics of spin weight $s=-2$ [28]. The sums are restricted to $\ell \geq 2$ and $-\ell \leq m \leq \ell$. With this ansatz for the Weyl scalar, the linearized Newman-Penrose equations lead to

$$
\left[r^{2} f \frac{d^{2}}{d r^{2}}-2(r-M) \frac{d}{d r}+U(r)\right] R_{\omega \ell m}(r)=T_{\omega \ell m},
$$

with

$$
U(r)=f^{-1}\left[(\omega r)^{2}-4 i \omega(r-3 M)\right]-(\ell-1)(\ell+2),
$$

and source term $T_{\omega \ell m}$ given in detail in Eq. (2.6) of [27].

We employ the Green's function method to obtain a solution of the previous equation. To build the Green's function, we need two linearly independent solutions of the homogeneous equation. We take $R_{\omega \ell m}^{H}$ and $R_{\omega \ell m}^{\infty}$. The latter one will describe purely outgoing waves escaping to infinity. The former one shall denote purely ingoing waves in the BH case (corresponding to a perfect absorber), and purely reflected waves in the ECO case (corresponding to a perfect mirror). For the considered ODE, the Wronskian of these two solutions must be a constant, and so it can be evaluated at any value of $r$. According the their definitions, these solutions should have the following asymptotic behaviors,

$$
\begin{gathered}
R_{\omega \ell m}^{H}(r \rightarrow+\infty) \sim \frac{1}{r} B_{\omega \ell m}^{\text {in }} e^{-i \omega r_{*}}+B_{\omega \ell m}^{\text {out }} r^{3} e^{i \omega r_{*}}, \\
R_{\omega \ell m}^{\infty}(r \rightarrow+\infty) \sim r^{3} e^{i \omega r_{*}},
\end{gathered}
$$

and the Wronskian yields

$$
\lim _{r \rightarrow \infty} \frac{R_{\omega \ell m}^{H} R_{\omega \ell m}^{\infty^{\prime}}-R_{\omega \ell m}^{H^{\prime}} R_{\omega \ell m}^{\infty}}{r^{2} f(r)}=2 i \omega B_{\omega \ell m}^{\mathrm{in}} .
$$

Here, the primes denote differentiation with respect to $r$. Thus, both solutions are linearly independent as long as $B_{\omega \ell m}^{\text {in }} \neq 0$ and $\omega \neq 0$. The Green's function is

$$
G\left(r, r^{\prime}\right)=\frac{\left[\theta\left(r^{\prime}-r\right) \frac{R_{\infty}\left(r^{\prime}\right) R_{H}(r)}{r^{\prime 2} f\left(r^{\prime}\right)}+\theta\left(r-r^{\prime}\right) \frac{R_{\infty}(r) R_{H}\left(r^{\prime}\right)}{r^{\prime 2} f\left(r^{\prime}\right)}\right]}{2 i \omega B_{\omega \ell m}^{\text {in }}} .
$$

Following the standard theory, we can write the inhomogeneous solution as

$$
\begin{aligned}
R_{\omega \ell m}(r)= & \int_{2 M}^{\infty} d r^{\prime} \frac{G\left(r, r^{\prime}\right) T_{\omega \ell m}\left(r^{\prime}\right)}{r^{\prime 2} f\left(r^{\prime}\right)} \\
& \sim \frac{r^{3} e^{i \omega r_{*}}}{2 i \omega B_{\omega \ell m}^{\mathrm{in}}} \int_{2 M}^{\infty} d r^{\prime} \frac{R_{\omega \ell m}^{H}\left(r^{\prime}\right) T_{\omega \ell m}\left(r^{\prime}\right)}{r^{\prime 4} f^{2}\left(r^{\prime}\right)},
\end{aligned}
$$

where in the last step we considered the limit $r \rightarrow \infty$.

\section{Energy flux}

To calculate the energy flux, it is helpful to introduce an auxiliary quantity $Z_{\ell m}$ by $R_{\omega \ell m}(r \rightarrow \infty)=$ $m_{0} Z_{\ell m} \delta(\omega-m \Omega) r^{3} e^{i \omega r_{*}}$. Then the flux formula reads

$$
\dot{E}_{s=2}=m_{0}^{2} \sum_{\ell m} \frac{\left|Z_{\ell m}\right|^{2}}{4 \pi \omega^{2}} .
$$

The auxiliary function can be calculated from the stressenergy tensor of a point particle orbiting around a Schwarzschild BH, and the result is [27]

$$
\begin{aligned}
Z_{\ell m}= & \frac{\pi}{i \omega r_{p}^{2} B_{\omega \ell m}^{\text {in }}}\left[\left({ }_{0} b_{\ell m}+2 i_{-1} b_{\ell m}\left[1+\frac{i \omega r_{p}}{2 f\left(r_{p}\right)}\right]-i_{-2} b_{\ell m}\left[1-\frac{M}{r_{p}}+\frac{i \omega r_{p}}{2}\right]\right) R_{\omega \ell m}^{H}\left(r_{p}\right)\right. \\
& \left.-\left(i_{-1} b_{\ell m}-_{-2} b_{\ell m}\left(1+\frac{i \omega r_{p}}{f\left(r_{p}\right)}\right)\right) r_{p} R_{\omega \ell m}^{H^{\prime}}\left(r_{p}\right)-\frac{1}{2}-2 b_{\ell m} r_{p}^{2} R_{\omega \ell m}^{H^{\prime \prime}}\left(r_{p}\right)\right],
\end{aligned}
$$

with coefficients 


$$
\begin{array}{r}
{ }_{0} b_{\ell m}=\frac{\sqrt{(\ell-1) \ell(\ell+1)(\ell+2)}}{2 \sqrt{1-\frac{3 M}{r_{p}}}}{ }_{0} Y_{\ell m}\left(\frac{\pi}{2}, 0\right), \\
{ }_{-1} b_{\ell m}=\sqrt{\frac{M(\ell-1)(\ell+2)}{r_{p}-3 M}}{ }_{-1} Y_{\ell m}\left(\frac{\pi}{2}, 0\right), \\
{ }_{-2} b_{\ell m}=\sqrt{\frac{M r_{p}^{2}}{r_{p}-3 M^{-2}}} Y_{\ell m}\left(\frac{\pi}{2}, 0\right) \Omega .
\end{array}
$$

From the identity ${ }_{s} Y_{\ell-m}\left(\frac{\pi}{2}, 0\right)={ }_{s} Y_{\ell m}\left(\frac{\pi}{2}, 0\right)(-1)^{s+\ell}$, it is easy to see that $Z_{\ell-m}=(-1)^{\ell} \bar{Z}_{\ell m}$. Consequently, the final expression for the energy flux is

$$
\dot{E}_{s=2}=m_{0}^{2} \sum_{\ell=2}^{\infty} \sum_{m=1}^{\ell} \frac{\left|Z_{\ell m}\right|^{2}}{2 \pi \omega^{2}}
$$

with $\omega=m \Omega$.

\section{Reducing the problem to solving the Regge-Wheeler equation}

Chandrasekhar [29] showed that if $X_{\omega \ell m}(r)$ is a solution to the Regge-Wheeler equation

$$
\left\{\frac{d^{2}}{d r_{*}^{2}}+\omega^{2}-V(r)\right\} X_{\omega \ell m}(r)=0
$$

with effective potential

$$
V(r)=f\left[\frac{\ell(\ell+1)}{r^{2}}-\frac{6 M}{r^{3}}\right],
$$

then

$$
\begin{aligned}
R_{\omega \ell m}(r)= & r^{3} V(r) X_{\omega \ell m}(r) \\
& +r^{3}\left[\frac{2}{r}\left(1-\frac{3 M}{r}\right)+2 i \omega\right]\left[\frac{\partial}{\partial r_{*}}+i \omega\right] X_{\omega \ell m}(r)
\end{aligned}
$$

is a solution to the homogeneous equation (B2). So rather than working with the ODE derived from the NewmanPenrose formalism, it is more convenient to solve the ReggeWheeler equation first and then apply the Chandrasekhar transformation to obtain the Weyl component $R_{\omega \ell m}^{H}(r)$ that governs the energy flux (B13).

The relevant solution $X_{\omega \ell m}^{H}(r)$ for our problem has the following asymptotic conditions, inherited from Eq. (B4):

$$
\begin{aligned}
X_{\omega \ell m}^{H}(r \rightarrow 2 M) & \sim A e^{-i \omega r_{*}}+B e^{i \omega r_{*}}, \\
X_{\omega \ell m}^{H}(r \rightarrow+\infty) & \sim A_{\omega \ell m}^{\text {in }} e^{-i \omega r_{*}}+A_{\omega \ell m}^{\text {out }} e^{i \omega r_{*}},
\end{aligned}
$$

where $[27,29]$

$$
\begin{gathered}
B_{\omega \ell m}^{\text {in }}=\frac{[12 i M \omega-(\ell-1) \ell(\ell+1)(\ell+2)]}{4 \omega^{2}} A_{\omega \ell m}^{\text {in }}, \\
B_{\omega \ell m}^{\text {out }}=-4 \omega^{2} A_{\omega \ell m}^{\text {out }} .
\end{gathered}
$$

As in the scalar case, for BHs, we shall fix $B=0$, while for ECOs, we have $B=-A x_{0}^{-2 i \omega}$.

\section{Some simplifications}

Although Eq. (B9) seems complicated at first, it can be further simplified. Recall that we consider the problem of having the particle far away from the BH so that $r_{p} \gg M$. Using the Kepler law $M \omega / m=M \Omega=\left(M / r_{p}\right)^{3 / 2}$, this automatically implies that $\varpi=2 M \omega \ll 1$. Inspection of Eqs. (B10)-(B12) shows that

$$
\begin{aligned}
{ }_{0} b_{\ell m} & \sim O\left(\varpi^{0}\right), \\
{ }_{-1} b_{\ell m} & \sim O\left(\varpi^{1 / 3}\right), \\
{ }_{-2} b_{\ell m} & \sim O\left(\varpi^{2 / 3}\right) .
\end{aligned}
$$

This means that $Z_{\ell m}$ in Eq. (B9) is dominated by the contribution involving ${ }_{0} b_{\ell m}$ unless ${ }_{0} Y_{\ell m}\left(\frac{\pi}{2}, 0\right)$ vanishes, which, according to Eq. (A36), happens when $\ell+m$ is an odd number. When this happens, $Z_{\ell m}$ is dominated by the term involving ${ }_{-1} b_{\ell m}$, but this is suppressed in our approximation. For a given $\ell$, therefore, the energy flux (B13) will be dominated by modes for which $\ell+m$ is even. The leading order expression for $Z_{\ell m}$ is then

$$
Z_{\ell m} \approx \frac{\pi}{i \omega r_{p}^{2} B_{\omega \ell m}^{\mathrm{in}}}{ }_{0} b_{\ell m} R_{\omega \ell m}^{H}\left(r_{p}\right) .
$$

All that remains now is to calculate $A_{\text {in }}(\omega)$ and $X_{\omega \ell m}^{H}(r)$. From Eqs. (B19) and (B16), we get $B_{\text {in }}(\omega)$ and $R_{\omega \ell m}^{H}$, respectively. Then finally, we will use Eq. (B24) to calculate Eq. (B13). For the purpose of calculating $A_{\text {in }}(\omega)$ and $X_{\omega \ell m}^{H}(r)$, we shall follow the strategy done in the scalar case closely.

\section{Matched asymptotic expansions}

The homogeneous Regge-Wheeler equation can be written in two equivalent forms,

$$
\begin{aligned}
& \frac{d^{2} X}{d r_{*}^{2}}+\left(\omega^{2}-f\left(\frac{\ell(\ell+1)}{r^{2}}+\left(1-s^{2}\right) f^{\prime} / r\right)\right) X=0, \\
& \left(r^{2} f\left[\frac{X}{r}\right]^{\prime}\right)^{\prime}+\left(\frac{r^{2}}{f} \omega^{2}-\ell(\ell+1)+s^{2} r f^{\prime}\right) \frac{X}{r}=0,
\end{aligned}
$$


where the primes stand for radial derivatives and $s=2$. The scalar case is recovered by taking $s=0$.

\section{a. Near-region solution}

We analyze first the regime in which $\omega \ll \frac{1}{r-2 M}$. Comparing Eq. (B26) with Eq. (A19), it is clear that we can recycle the results done for the scalar case, as long as we keep track of the new factor $s^{2}$. Equation (A20) now reads

$$
x\left(\frac{X}{r}\right)^{\prime \prime}+\left(\frac{X}{r}\right)^{\prime}+\left[\frac{\varpi^{2}}{x(1-x)^{4}}-\frac{\ell(\ell+1)}{(1-x)^{2}}+\frac{s^{2}}{1-x}\right] \frac{X}{r}=0,
$$

where here the primes are derivatives with respect to $x \equiv f$ and $\varpi \equiv \omega r_{H}$. Making the substitution $X / r=$ $x^{i \varpi}(1-x)^{\ell+\epsilon+1} F$, we find, after neglecting the $O\left(\varpi^{2}\right)$ and $O(\epsilon)$ terms,

$x(1-x) \partial_{x}^{2} F+(c-(a+b+1) x) \partial_{x} F-\left(a b-s^{2}\right) F=0$, with $a, b, c$ being the parameters from Eq. (A22). Now we want to reabsorb the $s^{2}$ term into these parameters. Define $a^{\prime}$ and $b^{\prime}$ by $a b-s^{2}=a^{\prime} b^{\prime}$ and $a+b=a^{\prime}+b^{\prime}$. Solving this system leads to

$$
\begin{aligned}
& a^{\prime}=\ell+\epsilon+3+i \varpi+O\left(\varpi^{2}\right), \\
& b^{\prime}=\ell+\epsilon-1+i \varpi+O\left(\varpi^{2}\right) .
\end{aligned}
$$

The general solution in the near region is then

$$
\begin{aligned}
X_{\omega \ell m}= & A x^{-i \varpi}(1-x)^{\ell+\epsilon} F\left(a^{\prime}-c+1, b^{\prime}-c+1,2-c, x\right) \\
& +B x^{i \varpi}(1-x)^{\ell+\epsilon} F\left(a^{\prime}, b^{\prime}, c, x\right) .
\end{aligned}
$$

To study the far-region behavior of the above solution, use again the transformation properties of hypergeometric functions (A24). Then we find the large $r$ behavior $(x \sim 1)$

$$
\begin{aligned}
X_{\omega \ell m} \sim & {\left[\frac{r_{H}}{r}\right]^{\ell} \Gamma(-1-2 \ell-2 \epsilon)\left(\frac{B \Gamma(1+2 i \varpi)}{\Gamma(-\ell-\epsilon+2+i \varpi) \Gamma(-\ell-\epsilon-2+i \varpi)}+\frac{A \Gamma(1-2 i \varpi)}{\Gamma(-\ell-\epsilon+2-i \varpi) \Gamma(-\ell-\epsilon-2-i \varpi)}\right) } \\
& +\left[\frac{r}{r_{H}}\right]^{\ell+1} \Gamma(1+2 \ell+2 \epsilon)\left(\frac{B \Gamma(1+2 i \varpi)}{\Gamma(\ell+\epsilon+3+i \varpi) \Gamma(\ell+\epsilon-1+i \varpi)}+\frac{A \Gamma(1-2 i \varpi)}{\Gamma(\ell+\epsilon+3-i \varpi) \Gamma(\ell+\epsilon-1-i \varpi)}\right) .
\end{aligned}
$$

\section{b. The far-region solution}

In the far region (i.e., when $r \gg r_{0}$ ), the wave equation (B25) reduces to

$$
\partial_{r}^{2} X+\left(\omega^{2}-\ell(\ell+1) / r^{2}\right) X=0,
$$

with solutions

$$
X_{\omega \ell m}=r^{1 / 2}\left(\alpha J_{\ell+1 / 2}(\omega r)+\beta J_{-\ell-1 / 2}(\omega r)\right),
$$

and for small $r$ reduces to

$$
X_{\omega \ell m} \sim \alpha \frac{\omega^{\ell+1 / 2}}{2^{\ell+1 / 2} \Gamma(\ell+3 / 2)} r^{\ell+1}+\beta \frac{2^{\ell+1 / 2}}{\omega^{\ell+1 / 2} \Gamma[-\ell+1 / 2]} r^{-\ell} .
$$

\section{c. Matching}

From the behavior of the Bessel functions at $r \rightarrow \infty$, the solution (B30) is asymptotic to

$$
X_{\omega \ell m} \sim e^{i\left(\omega r+\frac{\pi}{2} \ell\right)} \sqrt{\frac{2}{\pi \omega}} \frac{\beta-i \alpha(-1)^{\ell}}{2}+(i \rightarrow-i),
$$

and by demanding now the behavior (B18), we get

$$
A_{\omega \ell m}^{\text {in }}=\frac{e^{-i \ell \pi / 2}\left(\beta+i \alpha e^{i \ell \pi}\right)}{\sqrt{2 \pi \omega}} .
$$

Now we want to express $\alpha$ and $\beta$ in terms of the boundary parameters $A, B$. As in the scalar case, we proceed to match the small $r$ behavior of the far-region solution to the large $r$ behavior of the near-region solution.

First, we use the approximation [correct up to $\mathcal{O}(\varpi, \epsilon)$ ]

$$
\frac{\Gamma(-2 \ell-2 \epsilon-1)}{\Gamma(2-\ell-\epsilon+i \varpi)}=\frac{(-1)^{\ell+1} \Gamma(\ell-1)}{2 \Gamma(2 \ell+2)} .
$$

In addition, we can use 


$$
\begin{aligned}
\frac{\Gamma(1+2 i \varpi)}{\Gamma(-\ell-\epsilon+i \varpi)}= & \frac{\Gamma(1+2 i \varpi)(i \varpi-\epsilon)(-1)^{\ell}}{\Gamma(-\ell+i \varpi) \Gamma(\ell-\epsilon+\varpi i+1)} \\
& \times \prod_{k=1}^{\ell}\left[k^{2}-(i \varpi-\epsilon)^{2}\right]
\end{aligned}
$$

to show that, up to $\mathcal{O}\left(\varpi^{2}, \epsilon\right)$,

$$
\frac{\Gamma(1+2 i \varpi)}{\Gamma(-2-\ell-\epsilon+i \varpi)}=(-1)^{\ell} i \varpi \Gamma(\ell+3) .
$$

Using the above results, we find, in the limit $\varpi \ll 1$,

$$
\begin{aligned}
& \alpha \approx \frac{2^{\ell+1 / 2}}{\sqrt{r_{H}} \varpi^{\ell+1 / 2}} \frac{\Gamma(1+2 \ell) \Gamma(\ell+3 / 2)}{\Gamma(\ell+3) \Gamma(\ell-1)}(A+B), \\
& \beta \approx \frac{\varpi^{\ell+3 / 2} \Gamma(-\ell+1 / 2) \Gamma(\ell+3) \Gamma(\ell-1) i}{2^{\ell+3 / 2} \sqrt{r_{H}} \Gamma(2 \ell+2)}(A-B) .
\end{aligned}
$$

Putting these expressions into Eq. (B33), we find

$$
A_{\omega \ell m}^{\text {in }}=i \frac{(A+B) \Gamma(1+2 \ell) 4^{\ell+1} \Gamma(\ell+3 / 2) \Gamma(2 \ell+2)(-1)^{\ell}+(A-B) \Gamma(-\ell+1 / 2) \varpi^{2(\ell+1)} \Gamma(\ell+3)^{2} \Gamma(\ell-1)^{2}}{2^{\ell+2} i^{\ell} \sqrt{\pi} \varpi^{\ell+1} \Gamma(\ell+3) \Gamma(\ell-1) \Gamma(2 \ell+2)} .
$$

\section{The black hole flux formulas}

For BHs, $B=0$. Equation (B37) together with Eq. (B19) gives, in the approximation $\varpi \ll 1$,

$$
\left|B_{\omega \ell m}^{\text {in }}\right|^{2} \approx|A|^{2} \frac{\Gamma(1+2 \ell)^{2} 4^{\ell-2} \Gamma(\ell+3 / 2)^{2}}{\pi \omega^{4} \varpi^{2(\ell+1)} \Gamma(\ell-1)^{4}} .
$$

From Eq. (B16), the Weyl scalar function can be calculated as

$R^{H}\left(r_{p}\right) \approx r_{p} \ell(\ell+1) X\left(r_{p}\right)+\left.2 r_{p}^{2}\left(\frac{d}{d r}+i \omega\right) X\right|_{r_{p}}$,

where we notice that $\varpi=\left(M / r_{p}\right)^{3 / 2} \ll M / r_{p}$. To calculate the derivative we use Eq. (B27), and then we take the large distance limit with Eq. (B27). The large distance limit of $X_{\omega \ell m}$ is taken from Eq. (B28). Doing the calculation in detail, and staying always in the approximation in which $\varpi \ll 1$ (or equivalently $r_{p} \gg r_{H}$ ), we end up with

$$
\begin{gathered}
X\left(r_{p}\right) \approx\left(\frac{r_{p}}{r_{H}}\right)^{\ell+1} A \frac{\Gamma(1+2 \ell)}{\Gamma(\ell+3) \Gamma(\ell-1)}, \\
\left.\frac{d}{d r} X\right|_{r_{p}} \approx\left(\frac{r_{p}}{r_{H}}\right)^{\ell+1} \frac{A}{r_{p}} \frac{(\ell+1) \Gamma(1+2 \ell)}{\Gamma(\ell+3) \Gamma(\ell-1)} .
\end{gathered}
$$

Then it is straightforward to write from Eq. (B39)

$$
R_{\omega \ell m}^{H}\left(r_{p}\right) \approx\left(\frac{r_{p}}{r_{H}}\right)^{\ell+1} A r_{p} \frac{\Gamma(1+2 \ell)}{\Gamma(\ell+1) \Gamma(\ell-1)} .
$$

From Eqs. (B24) and (B10) and using Eq. (A36) for odd $m+\ell$, we get, after several simplifications,

$$
\left|Z_{\ell m}\right|^{2} \approx \frac{m^{2 \ell+4} M^{\ell+2}}{r_{p}^{\ell+6}} \frac{4 \pi \Gamma(\ell+3) \Gamma(\ell-1)}{\Gamma(2 \ell+2) \Gamma(\ell+1)^{2}}
$$

and the energy flux (B13) is finally

$$
\dot{E}_{s=2} \approx m_{0}^{2} \sum_{\ell=2}^{\infty} \sum_{m=\mathrm{even}}^{\ell} \frac{m^{2 \ell+2} M^{\ell+1}}{r_{p}^{\ell+3}} \frac{2 \Gamma(\ell+3) \Gamma(\ell-1)}{\Gamma(2 \ell+2) \Gamma(\ell+1)^{2}} .
$$

Note that for the leading order contribution, $\ell=m=2$, we get

$$
\dot{E}_{s=2} \approx \frac{32}{5} \frac{m_{0}^{2} M^{3}}{r_{p}^{5}}=\frac{32}{5}\left(\frac{m_{0}}{M}\right)^{2}(M \Omega)^{10 / 3},
$$

which agrees with the well-known Einstein quadrupole formula.

\section{The QNMs of ECOs}

Following the same reasoning as in the scalar case, we derive the QNM frequencies as the roots of $A_{\omega \ell m}^{\text {in }}=0$. By setting Eq. (B37) equal to zero, we can write

$$
e^{i 2 \varpi \log x_{0}} R(\varpi)=1, \quad R(\varpi)=\frac{1-F_{s=2}(\ell) \varpi^{2 \ell+2}}{1+F_{s=2}(\ell) \varpi^{2 \ell+2}},
$$

now with

$F_{s=2}(\ell)=\frac{\Gamma(1 / 2-\ell) \Gamma(\ell+3)^{2} \Gamma(\ell-1)^{2}(-1)^{\ell+1}}{4^{\ell+1} \Gamma(\ell+3 / 2) \Gamma(2 \ell+1) \Gamma(2 \ell+2)}$.

We solve the equation iteratively by solving $e^{i 2 \varpi_{i+1} \log x_{0}} R\left(\varpi_{i}\right)=1$ for $\varpi_{i+1}$, with the initial condition $\varpi_{0}=0$. The solution is given in Eq. (A42), but with the new $F_{s=2}$. After some simplifications, the result for the quasinormal modes of gravitational perturbations is

$$
\varpi_{s=2} \simeq \frac{n \pi}{\log x_{0}}-i \frac{(2 n \pi)^{2 \ell+2} \Gamma(\ell+1)^{2} \Gamma(\ell-1)^{2} \Gamma(\ell+3)^{2}}{8\left|\log x_{0}\right|^{2 \ell+3}(2 \ell+1) \Gamma(2 \ell+1)^{3} \Gamma(2 \ell+2)} .
$$


Again, the nonvanishing imaginary part has the correct sign and takes into account the exponential decay of the mode in time (stability).

\section{The ECO flux formulas}

For ECOs, we have $B=-A x_{0}^{-2 i \varpi}$. Equation (B37) yields

$$
\begin{aligned}
& \left|A_{\text {in }}(\omega)\right|^{2} \\
& \quad \approx|A|^{2} \frac{\Gamma(1+2 \ell)^{2} 4^{2 \ell+2} \Gamma(\ell+3 / 2)^{2} \Gamma(2 \ell+2)^{2} \sin ^{2}\left(\varpi \log x_{0}\right)+\Gamma(-\ell+1 / 2)^{2} \varpi^{4(\ell+1)} \Gamma(\ell+3)^{4} \Gamma(\ell-1)^{4} \cos ^{2}\left(\varpi \log x_{0}\right)}{4^{\ell+1} \pi \varpi^{2(\ell+1)} \Gamma(\ell+3)^{2} \Gamma(\ell-1)^{2} \Gamma(2 \ell+2)^{2}},
\end{aligned}
$$

where we neglected the imaginary part of $\varpi^{2 \ell+2}$.

In order to get the energy flux, we need to calculate now (B16). First, the solution (B27) reads

$X_{\omega \ell m}(x)=A(1-x)^{\ell} x^{-i \varpi}\left[F(\ell+3-i \varpi, \ell-1-i \varpi, 1-2 i \varpi, x)-\frac{x^{2 i \varpi}}{x_{0}^{2 i \varpi}} F(\ell+3+i \varpi, \ell-1+i \varpi, 1+2 i \varpi, x)\right]$,

and from Eq. (B28), we find the asymptotic behavior to be dominated by

$$
X\left(r_{p}\right)=A\left[\frac{r_{p}}{r_{H}}\right]^{\ell+1} \Gamma(1+2 \ell)\left(\frac{1-x_{0}^{-2 i \varpi}}{\Gamma(-1+\ell) \Gamma(3+\ell)}+\frac{i\left(2 \gamma_{\mathrm{EM}}+\psi(-1+\ell)+\psi(3+\ell)\right)\left(1+x_{0}^{-2 i \varpi}\right) \varpi}{\Gamma(\ell-1) \Gamma(3+\ell)}\right)+\ldots
$$

where the ellipsis denotes higher order terms in $r_{H} / r_{p}$ and $\varpi$. Because $\varpi \approx \frac{n \pi}{\mid \log x_{0}}$, the first term in the parentheses is clearly subdominant, and we shall neglect it. In the BH case, this would have been the dominant contribution, but because of the different boundary conditions now chosen, it is the subsequent leading term that dominates now.

On the other hand, we calculate the derivative from Eq. (B47),

$$
\begin{aligned}
\left.\frac{d}{d r} X\right|_{r_{p}}= & \frac{2 M}{r_{p}^{2}}\left[-X\left(\frac{\ell}{1-x}+\frac{i \varpi}{x}\right)-2 i \varpi A(1-x)^{\ell} \frac{x^{i \varpi-1}}{x_{0}^{2 i \varpi}} F(\ell+3+i \varpi, \ell-1+i \varpi, 1+2 i \varpi, x)\right. \\
& \left.+A(1-x)^{\ell} x^{-i \varpi}\left(\frac{(\ell+3-i \varpi)(\ell-1-i \varpi)}{1-2 i \varpi} F(\ell+4-i \varpi, \ell-i \varpi, 2-2 i \varpi, x)-\frac{x^{2 i \varpi}}{x_{0}^{2 i \varpi}} \text { c.c. }\right)\right],
\end{aligned}
$$

and analyze the large distance behavior using Eq. (A24). Taking care of the issues commented upon above, the leading order contribution is

$$
\left.\frac{d}{d r} X\right|_{r_{p}}=\frac{2 M}{r_{p}^{2}} A\left[\frac{r_{p}}{r_{H}}\right]^{\ell+2} \frac{2 i\left(2 \gamma_{\mathrm{EM}}+\psi(-1+\ell)+\psi(3+\ell)\right) \varpi}{\Gamma(\ell-1) \Gamma(3+\ell)} \Gamma(1+2 \ell)(1+\ell)+\ldots
$$

Consequently, from Eq. (B39), we get

$$
R_{\omega \ell m}^{H} \approx(\ell(\ell+1)+1) 2 r_{H} A\left[\frac{r_{p}}{r_{H}}\right]^{l+2} \frac{2 i\left(2 \gamma_{\mathrm{EM}}+\psi(-1+\ell)+\psi(3+\ell)\right) \varpi}{\Gamma(\ell-1) \Gamma(3+\ell)} \Gamma(1+2 \ell) .
$$

Finally, from Eqs. (B24), (B10), (B19), and (A36), we get the energy flux after several simplifications:

$\left|Z_{\ell m}\right|^{2} \approx \frac{4^{2} \pi^{2} \varpi^{2 \ell+6} r_{p}^{2 \ell} r_{H}^{-2 \ell-4} \Gamma(2 \ell+2)^{3} \Gamma(2 \ell+1)^{2} \Gamma(\ell-1)(\ell+1)^{4}\left[2 \gamma_{\mathrm{EM}}+\psi(\ell-1)+\psi(\ell+3)\right]^{2} / \Gamma(\ell+1)^{2} / \Gamma(\ell+3)}{\Gamma(1+2 \ell)^{2} 4^{2 \ell+2} \Gamma(\ell+3 / 2)^{2} \Gamma(2 \ell+2)^{2} \sin ^{2}\left(\varpi \log x_{0}\right)+\Gamma(-\ell+1 / 2)^{2} \varpi^{4(\ell+1)} \Gamma(\ell+3)^{4} \Gamma(\ell-1)^{4} \cos ^{2}\left(\varpi \log x_{0}\right)}$.

Near the QNM frequencies, this is 


$$
\dot{E}_{s=2} \approx \frac{m_{0}^{2}}{M^{2}} \frac{m^{4 \ell / 3}}{(n \pi)^{10 \ell / 3}}(\log \epsilon)^{10 \ell / 3} \frac{2 \pi \Gamma(2 \ell+2)^{3} \Gamma(2 \ell+1)^{2}(\ell+1)^{4}\left[2 \gamma_{\mathrm{EM}}+\psi(\ell-1)+\psi(\ell+3)\right]^{2}}{2^{2 \ell / 3} \Gamma(-\ell+1 / 2)^{2} \Gamma(\ell+3)^{5} \Gamma(\ell-1)^{3} \Gamma(\ell+1)^{2}} .
$$

\section{The resonance width}

We follow the same method as in the scalar case. Assuming the frequency band to be narrow around the resonance modes, we can expand as in Eq. (A48). On the other hand, the gravitational flux (B51) can be written as

$$
\dot{E}_{s=2}(r)=\frac{a_{\ell m} \varpi^{2 \ell / 3+4}(r)}{b_{\ell} \varpi^{4 \ell+4}(r) \cos ^{2}\left(\varpi(r) \log x_{0}\right)+c_{\ell} \sin ^{2}\left(\varpi(r) \log x_{0}\right)},
$$

now with $b_{\ell} \equiv \Gamma(-\ell+1 / 2)^{2} \Gamma(\ell-1)^{4} \Gamma(\ell+3)^{4}>0$ and $c_{\ell} \equiv 4^{2 \ell+2} \Gamma(2 \ell+2)^{2} \Gamma(2 \ell+1)^{2} \Gamma(\ell+3 / 2)^{2}>0\left(a_{\ell m}\right.$ will not be needed). The flux peaks at $r_{\mathrm{QNM}}$ and reads $\dot{E}_{s=2}\left(r_{\mathrm{QNM}}\right)=\frac{a_{\ell m}}{b_{\ell}} \varpi_{\mathrm{QNM}}^{-10 / 3 \ell}$. By expanding the energy flux up to second order, we get

$$
\frac{\dot{E}_{s=2}(r)}{\dot{E}_{s=2}\left(r_{\mathrm{QNM}}\right)}=\left[1+5 \ell \frac{r-r_{\mathrm{QNM}}}{r_{\mathrm{QNM}}}+\left(\frac{r-r_{\mathrm{QNM}}}{r_{\mathrm{QNM}}}\right)^{2}\left(\frac{9 n^{2} \pi^{2}-35 \ell+50 \ell^{2}}{4}-\frac{9 c_{\ell} n^{2} \pi^{2} \varpi_{0}^{-4-4 \ell}}{4 b_{\ell}}\right)\right]+\ldots
$$

Demanding now $\dot{E}_{s=2}(r)=\frac{1}{2} \dot{E}_{s=2}\left(r_{\mathrm{QNM}}\right)$, one gets a quadratic equation for $\left(r-r_{\mathrm{QNM}}\right) / r_{\mathrm{QNM}} \equiv y$,

$y^{2}\left[\frac{9 n^{2} \pi^{2}-35 \ell+50 \ell^{2}}{4}-\frac{9 c_{\ell} n^{2} \pi^{2} \varpi_{\mathrm{QNM}}^{-4-4 \ell}}{4 b_{\ell}}\right]+y(5 \ell)=-\frac{1}{2}$,

which again leads to two solutions, $r_{+}$and $r_{-}$, corresponding to the outer and inner radii of the width. The solution, in the approximation in which $\varpi_{\mathrm{QNM}} \ll 1$ [recall Eq. (B46)], takes the simple form

$$
y_{ \pm} \approx \pm \frac{\varpi_{\mathrm{QNM}}^{2 \ell+2}}{k \pi} \sqrt{\frac{2 b_{\ell}}{9 c_{\ell}}} .
$$

Using Eq. (B45), it can be checked that $\sqrt{b_{\ell} / c_{\ell}}=$ $F_{s=2}(\ell)(-1)^{\ell+1}$. Thus, we finally arrive at

$$
\begin{aligned}
\left|\frac{r_{+}-r_{-}}{r_{\mathrm{QNM}}}\right| & \approx \sqrt{\frac{8}{9}} \frac{\operatorname{Im} \varpi_{\mathrm{QNM}}}{\operatorname{Re} \varpi_{\mathrm{QNM}}} \\
& \approx \sqrt{\frac{8}{9}} \frac{(2 n \pi)^{2 \ell+1} \Gamma(\ell+1)^{2} \Gamma(\ell-1)^{2} \Gamma(\ell+3)^{2}}{4\left|\log x_{0}\right|^{2 \ell+2}(2 \ell+1) \Gamma(2 \ell+1)^{3}}
\end{aligned}
$$

where we used the form of Eq. (A42). The width in frequency follows the same approach as in Eq. (A51), yielding the same relation.
[1] V. Cardoso and P. Pani, Living Rev. Relativity 22, 4 (2019).

[2] V. Baibhav et al., arXiv:1908.11390.

[3] S. A. Hughes, Phys. Rev. D 64, 064004 (2001); 88, 109902(E) (2013).

[4] N. V. Krishnendu, K. G. Arun, and C. K. Mishra, Phys. Rev. Lett. 119, 091101 (2017).

[5] V. Cardoso, E. Franzin, A. Maselli, P. Pani, and G. Raposo, Phys. Rev. D 95, 084014 (2017).

[6] A. Maselli, P. Pani, V. Cardoso, T. Abdelsalhin, L. Gualtieri, and V. Ferrari, Phys. Rev. Lett. 120, 081101 (2018).

[7] N. Sennett, T. Hinderer, J. Steinhoff, A. Buonanno, and S. Ossokine, Phys. Rev. D 96, 024002 (2017).

[8] N. V. Krishnendu, C. K. Mishra, and K. G. Arun, Phys. Rev. D 99, 064008 (2019).
[9] S. Datta and S. Bose, Phys. Rev. D 99, 084001 (2019).

[10] V. Cardoso, E. Franzin, and P. Pani, Phys. Rev. Lett. 116, 171101 (2016); 117, 089902(E) (2016).

[11] V. Cardoso, S. Hopper, C. F. B. Macedo, C. Palenzuela, and P. Pani, Phys. Rev. D 94, 084031 (2016).

[12] V. Cardoso and P. Pani, Nat. Astron. 1, 586 (2017).

[13] J. Abedi, H. Dykaar, and N. Afshordi, Phys. Rev. D 96, 082004 (2017).

[14] R. S. Conklin, B. Holdom, and J. Ren, Phys. Rev. D 98, 044021 (2018).

[15] K. W. Tsang, M. Rollier, A. Ghosh, A. Samajdar, M. Agathos, K. Chatziioannou, V. Cardoso, G. Khanna, and C. Van Den Broeck, Phys. Rev. D 98, 024023 (2018). 
[16] R. K. L. Lo, T. G. F. Li, and A. J. Weinstein, Phys. Rev. D 99, 084052 (2019).

[17] A. B. Nielsen, C. D. Capano, O. Birnholtz, and J. Westerweck, Phys. Rev. D 99, 104012 (2019).

[18] N. Uchikata, H. Nakano, T. Narikawa, N. Sago, H. Tagoshi, and T. Tanaka, Phys. Rev. D 100, 062006 (2019).

[19] K. W. Tsang, A. Ghosh, A. Samajdar, K. Chatziioannou, S. Mastrogiovanni, M. Agathos, and C. V. D. Broeck, arXiv: 1906.11168.

[20] C. F. B. Macedo, P. Pani, V. Cardoso, and L. C. B. Crispino, Astrophys. J. 774, 48 (2013).

[21] P. Pani, E. Berti, V. Cardoso, Y. Chen, and R. Norte, Phys. Rev. D 81, 084011 (2010).

[22] E. Berti, V. Cardoso, and A. O. Starinets, Classical Quantum Gravity 26, 163001 (2009).
[23] E. Maggio, V. Cardoso, S. R. Dolan, and P. Pani, Phys. Rev. D 99, 064007 (2019).

[24] E. Poisson and M. Sasaki, Phys. Rev. D 51, 5753 (1995).

[25] V. Cardoso, O. J. C. Dias, J. P. S. Lemos, and S. Yoshida, Phys. Rev. D 70, 044039 (2004); 70, 049903(E) (2004).

[26] M. Abramowitz and I. A. Stegun, Handbook of Mathematical Functions with Formulas, Graphs, and Mathematical Tables (Dover, New York, 1972).

[27] E. Poisson, Phys. Rev. D 47, 1497 (1993).

[28] J. N. Goldberg, A. J. MacFarlane, E. T. Newman, F. Rohrlich, and E. C. G. Sudarshan, J. Math. Phys. (N.Y.) 8, 2155 (1967).

[29] S. Chandrasekhar, The Mathematical Theory of Black Holes (Clarendon Press, Oxford, 1992). 